\title{
Universalidad y aplicabilidad de las matemáticas en Wittgenstein y el empirismo logicista
}

\author{
Axel Arturo Barceló Aspeitia
}

\section{I}

\section{ntroducción}

De acuerdo a un gran número de recuentos históricos de su vida, Wittgenstein regresó a Cambridge en 1929 con el modesto objetivo de corregir algunos detalles del Tractatus Logico-Philosophicus, no para empezar un nuevo periodo en la evolución de su pensamiento ni para desarrollar un nuevo sistema filosófico. ${ }^{1}$ Todo parece indicar que Wittgenstein regresó a la vida académica bajo la impresión de que el proyecto filosófico planteado en el Tractatus seguía incompleto, y que aún quedaban problemas sin resolver. Sin embargo, conforme trabajaba sobre este objetivo, pronto se dio cuenta de que lo que se requería era una transformación profunda y radical del proyecto original. En consecuencia, su pensamiento filosófico experimentó una rápida evolución durante los primeros años de la década de los treintas.

$\mathrm{Al}$ igual que durante el resto de su carrera filosófica, esta nueva etapa estuvo fuertemente dominada por una preocupación por los fundamentos de las matemáticas. Este nuevo periodo fue tanto una continuación de las ideas y preocupaciones que guiaron su trabajo anterior, como una innovación radical de ese pensamiento. La hipótesis central que Wittgenstein exploró duran-

${ }^{1}$ G. H. von Wright y Brian McGuiness, eds., "Introduction", en Ludwig Wittgenstein, Cambridge Letters: Correspondence with Russell, Keynes, Moore, Ramsey and Sraffa. Oxford, Blackwell, 1995, p. 4. G. E. Moore, sin embargo, no concurre. En un pasaje introductorio a "Wittgenstein's Lectures in 1930-1933", escribe: "La afirmación que aparece en su obituario del periódico The Times del 2 de mayo de 1951, según la cual [Wittgenstein] llegó a Cambridge en 1929 "para una visita corta" se aleja mucho de la verdad". [The statement in the Obituary notice in The Times for May 2, 1951, that he arrived in Cambridge in 1929 "for a short visit" is very far from the truth]. Robert R. Ammerman, ed., Classics of Analytic Philosophy. Nueva York, McGraw-Hill, 1965, p. 234 , nota 1 . 
te estos años fue la de ver a las matemáticas como parte integral de la gramática del lenguaje. Sería imposible tratar de explicar en unas cuantas páginas el original carácter de la filosofía de las matemáticas de Wittgenstein durante este periodo. El objetivo de este artículo es mucho más modesto: explicar cómo Wittgenstein articula la hipótesis de las matemáticas como gramática para ofrecer una nueva explicación de la aplicación de las matemáticas, en contraposición a la historia tradicional dada por el logicismo y el empirismo lógico. La aportación principal de esta nueva manera de entender la aplicación de las matemáticas es que explícitamente evita el extendido mito de las matemáticas como ciencia universal.

El objetivo de este artículo puede entenderse a varios niveles. Su objetivo exegético es interpretar ciertos pasajes de la obra intermedia de Wittgenstein. En particular, me centro en un pasaje que ocurre tanto en el parágrafo 15 de la parte II, sección III de la Gramática filosófica,${ }^{2}$ como en el parágrafo 111 de las Observaciones filosóficas. ${ }^{3}$ Este breve pasaje es singularmente importante dentro de la obra wittgensteineana, pues en él se encuentra concentrado el corazón de la teoría de Wittgenstein de la aplicación de las matemáticas. En él, Wittgenstein destila su crítica al empirismo lógico y el logicismo, respecto a este problema tan importante dentro de la filosofía de las matemáticas. Además, aporta un argumento importante para separar las nociones de analiticidad, necesidad y universalidad, respecto a las proposiciones matemáticas.

Durante los primeros años de la década de los treintas, Wittgenstein explora su propuesta de matemáticas como gramática en busca de una alternativa a la posición de autores como Russell, Whitehead y él mismo en su obra temprana. Para entender la radical naturaleza de la alternativa wittgensteineana es necesario tener una imagen clara de la propuesta a la que se opone. En este artículo, llamo a esta posición empirismo logicista, pues es una síntesis del empirismo lógico inglés con el logicismo centroeuropeo. Es por ello que el artículo está estructurado en tres partes: una parte introductoria y otras dos partes cada una dedicada a los tratamientos del problema de la aplicación de las matemáticas del empirismo logicista y el Wittgenstein del periodo intermedio, respectivamente. En la primera sección introduzco el problema de la aplicación de las matemáticas tal y como aparece en la obra intermedia de

${ }^{2}$ Ludwig Wittgenstein, Gramática filosófica (Philosophische Grammatik). Ed. bilingüe. Trad. de Luis Felipe Segura del texto editado por Rush Rhees. México, UnAM, Instituto de Investigaciones Filosóficas, 1992. (Philosophical Grammar. Ed. de Rush Rhees. Trad. de Anthony Kenny. Berkeley, Universidad de California, 1974).

${ }^{3}$ L. Wittgenstein, Observaciones filosóficas (Philosophische Bemerkungen). Ed. bilingüe. Trad. de Alejandro Tomasini. México, UNAM, Instituto de Investigaciones Filosóficas, 1988. [Philosophical Remarks. Ed. de Rush Rhees. Trad. de Raymond Hargeaves y Roger White. Oxford, Basil Blackwell, 1975]. 
Wittgenstein. En las siguientes dos secciones explico cómo se entiende y cómo se resuelve este problema dentro del empirismo logicista. En la tercera sección me concentro en la obra de Bertrand Russell, pues es claro que Wittgenstein le dirigió sus comentarios en este respecto. Finalmente, las últimas dos secciones están dedicadas a la crítica de Wittgenstein a la solución del empirismo logicista, y a su propia propuesta de solución, respectivamente.

\title{
La aplicación del cálculo en la solución de problemas prácticos
}

\author{
En este momento podemos decir: la aritmética \\ es su propia aplicación. El sistema de cálculo
} es su propia aplicación. ${ }^{4}$

El tema central de la sección III, "Fundamentos de las matemáticas..." [Grundlagen der Mathematik...], de la parte II, "Acerca de la lógica y las matemáticas" [Über Logik und Mathematik] de la Gramática filosófica [Philosophische Grammatik] de Wittgenstein es cómo se aplica la matemática, la aritmética de números naturales en particular, ${ }^{5}$ en la solución de problemas prácticos. El resultado de sus observaciones se encuentra compactamente sintetizado en el parágrafo 15 de la susodicha sección, donde Wittgenstein escribe:

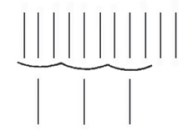

Suponga que yo quisiera resolver el siguiente problema con este cálculo: si tengo 11 manzanas y quiero repartirlas entre un grupo de personas de tal manera que cada una reciba 3 manzanas, ¿a cuántas personas puedo repartir? El cálculo me da la solución 3. Ahora suponga que realizo la distribución y que al final hubiera cuatro personas, cada una con tres manzanas en la mano. ¿Diría entonces que el cálculo ha dado un resultado falso? Por supuesto que no. Y eso significa únicamente que el cálculo no era un experimento.

\footnotetext{
4 "Hier kann man nun sagen: Die Arithmetik ist ihre eigene Anwendung. Der Kalkül ist seine eigene Anwendung" (L. Wittgenstein, Gramática filosófica, parágrafo 15).

${ }^{5}$ Aunque Wittgenstein también considera ejemplos de geometría [626, 628] y teoría de conjuntos [620].
} 
Podría parecer como si el cálculo matemático nos justificara a hacer una predicción, digamos, la de que podría darle a tres personas su porción y quedarían 2 manzanas. Pero no es así. Lo que nos justifica el hacer esta predicción es una hipótesis física, la cual se ubica fuera del cálculo. El cálculo es sólo un estudio de formas lógicas, de estructuras, y por sí mismo no puede producir nada nuevo. ${ }^{6}$

Uno de los focos de interés de Wittgenstein dentro de la filosofía de las matemáticas es el papel que juegan los cálculos matemáticos en la solución de problemas, tanto dentro como fuera de las matemáticas. ${ }^{7}$ En particular, Wittgenstein está interesado en saber si los cálculos matemáticos juegan el mismo papel en la solución de ambos tipos de problemas. ${ }^{8}$ Para resaltar este problema, Wittgenstein pone especial cuidado en distinguir entre el resultado (Resultät) de un cálculo, y la solución (Lösung) de un problema práctico. ${ }^{9}$

6 "Angenommen, mit dieser Rechnung wollte ich folgende Aufgabe lösen: Wenn ich 11 Äpfel habe und Leute mit je 3 Äpfeln beteilen will, wie viele Leute kann ich beteilen? Die Rechnung liefert mir die Lösung 3. Angenommen nun, ich vollzöge alle Handlugen des Beteilens und am Ende hätten 4 Personen je 3 Äpfel in der Hand. Würde ich nun sagen, die Ausrechnung hat ein falsches Resultat ergeben? Natürlich nicht. Und das heißt ja nur, daß die Ausrechnung kein Experiment war.

Es könnte scheinen, als berechtigte uns die mathematische Ausrechnung zu einer Vorhersagung, etwa, daß ich 3 Personen werde beteilen können und 2 Äpfel übrigbleiben werden. So ist es aber nicht. Zu dieser Vorhersagung berechtigt uns eine physicalische Hypothese, die Außerhalb der Rechnung steht. Die Rechnung ist nur eine Betrachtung der logischen Formen, der Strukturen, und kann an sich nicht Neues liefern" (L. Wittgenstein, Gramática filosófica, p. 602. Cf. L. Wittgenstein, Observaciones filosóficas, parágrafo 111, p. 122).

${ }^{7}$ La distinción corresponde a problemas internos a las matemáticas que pueden resolverse con la mera resolución de un cálculo y problemas externos que requieren la aplicación de esta solución para hacer algo fuera del cálculo. Para estos últimos, Wittgenstein usa la palabra alemana Aufgabe, la cual contiene tanto el sentido de "problema" como el de "tarea". Es por ello que, en este contexto, Aufgabe es comúnmente traducido como "problema práctico". En este artículo uso las expresiones "problemas de cálculo" y "problemas prácticos" para referirme a estos dos tipos de problemas. Prefiero reservar los términos "matemática pura" y "matemática aplicada" para distinguir diferentes tipos de lenguaje, en vez de diferentes tipos de problemas.

${ }^{8}$ Este problema conecta de manera directa con su interés en distinguir entre cálculo y experimento; ley matemática y ley natural.

${ }^{9}$ Desafortunadamente, nuestro español no distingue entre el cálculo como un sistema de reglas matemáticas (Kalkül) y el cálculo (Rechnung) como acción y efecto de calcular (Rechnen). Wittgenstein, en contraste, contaba con tal distinción dentro del alemán. Por lo tanto, en este texto, utilizo "sistema (matemático) de cálculo" para traducir el término alemán Kalkül y "cálculo" para traducir Rechnung. De esta manera, puedo distinguir entre un cálculo como $3+14=17$ y un sistema de cálculo como la aritmética de los números naturales. 
En ciertos casos, como el del ejemplo en este pasaje, el resultado de un cálculo matemático es también la solución de un problema práctico. En este ejemplo, el número 3 es tanto el resultado del cálculo como la solución al problema. El mismo número juega ambos roles. Pero los roles, en ellos mismos, son muy distintos. Lo que llama la atención a Wittgenstein de este tipo de casos es la capacidad que tienen los números y, en general, las entidades matemáticas, de jugar ambos roles, es decir, la capacidad de las entidades matemáticas de trascender los límites del cálculo para obtener vida fuera del ámbito de las matemáticas puras. Para Wittgenstein, esta doble dimensión de los números yace al centro del problema filosófico de la aplicación de la aritmética.

Para Wittgenstein, al establecer la posibilidad de hacer predicciones extra-matemáticas, un cálculo matemático puede dar solución a problemas que no sean puramente matemáticos. En este caso en particular, por ejemplo, el cálculo establece la posibilidad de predecir la distribución de once manzanas entre tres personas. Sin embargo, nos advierte Wittgenstein, si bien el cálculo establece la posibilidad de la predicción, no garantiza su verdad. La predicción puede ser, de hecho, falsa, en tanto que tiene sentido "suponer ahora, que yo fuera a llevar a cabo todo el proceso de distribución y al final quedaran 4 personas con 3 manzanas en la mano cada una". Es posible conjeturar que la predicción fallará. No se puede calcular el éxito de la predicción, sólo su posibilidad. La garantía de la predicción, si existe, debe yacer fuera del sistema matemático de cálculo. Las predicciones son hipótesis físicas externas a las matemáticas y sus sistemas de cálculo. Una línea muy clara separa a estos dos espacios lógicos: el del cálculo matemático y el de las predicciones físicas. El problema de la aplicación para la filosofía de las matemáticas es explicar cómo las entidades matemáticas pueden vivir en ambos espacios, es decir, cómo los resultados de uno tienen aplicación en el otro.

\section{El reto de la aplicación de las matemáticas al empirismo logicista}

Si bien el problema de la aplicación es central a toda filosofía de las matemáticas, representa un reto mayor al empirismo lógico de logicistas como Russell, Whitehead y el primer Wittgenstein. La fuente principal de esta dificultad es la logicización de la epistemología que ocurre con la síntesis del empirismo lógico y el logicismo. Esta logicización de la epistemología se manifiesta de diversas maneras. Para los objetivos de este artículo es suficiente traer a colación dos principios básicos del empirismo logicista. El primero de ellos es la identificación de la dualidad epistemológica entre conocimiento a priori y a posteriori y la distinción semántica entre enunciados analíticos y sintéticos. El segundo es 
la tesis de que las relaciones epistémicas de justificación deben descansar sobre relaciones de inferencia lógica entre proposiciones. Veamos con más detalle cómo estos dos principios definen el marco en el que la aplicabilidad de las matemáticas se vuelve problemática para el empirismo logicista.

Desde el trabajo de fundamentación logicista de las matemáticas de Gottlob Frege, el logicismo se había introducido como un rechazo a la tesis kantiana de las matemáticas como sintéticas y a priori. Para Frege, como para el resto de los logicistas, era absurdo pensar en proposiciones sintéticas que pudieran ser conocidas a priori o proposiciones analíticas que no lo fueran. ${ }^{10}$ Dentro de estas últimas, se contaban las proposiciones de la lógica y las matemáticas. Para el empirismo logicista, en consecuencia, la distinción entre el espacio lógico del cálculo matemático y el espacio de las predicciones físicas es primariamente epistemológica, ya que distingue entre dos campos de conocimiento, radicalmente diferentes. Por un lado, el conocimiento matemático es puramente lógico y a priori, mientras que el conocimiento del mundo físico es empírico y a posteriori. En estos términos, el problema de la aplicación de las matemáticas es el problema de comunicar el espacio de lo lógico y a priori con el espacio de lo empírico y a posteriori.

De acuerdo al segundo principio antes mencionado, dentro del empirismo logicista, las relaciones epistémicas sobrevienen sobre relaciones lógicas. En otras palabras, para que una proposición verdadera justifique epistémicamente la verdad de otra, debe existir una relación de consecuencia lógica a través de la cual la justificación se transmita de una a la otra. ${ }^{11}$ En el caso que nos ocupa en este momento, esto significa que para que el espacio del cálculo matemático se comunique con el de las predicciones físicas, es necesario que existan relaciones lógico-epistémicas entre proposiciones pertenecientes a cada uno de estos espacios.

Como consecuencia de estos dos principios, para el empirismo logicista, la aplicación de las matemáticas involucra el inferir predicciones físicas de

${ }^{10}$ Sin embargo, y en sentido estricto, las nociones de juicio analítico y sintético de los logicistas no son las mismas que las de Kant. En Los fundamentos de la aritmética, Frege argumenta en contra de la formulación kantiana, y la sustituye por otra cuya correspondencia con la distinción epistemológica a priori/a posteriori es directa y explícita (Cf. Gottlob Frege, Die Grundlagen der Arithmetik. Breslau, Wilhelm Koebner, 1884, parágrafos 12 y 87).

${ }^{11}$ Así, las teorías se organizan lógicamente de tal manera que la justificación de cada proposición de una teoría depende de la justificación de las proposiciones básicas que forman sus fundamentos y de la coherencia y consistencia lógica de la teoría necesarias para transmitir esta justificación epistémica desde los fundamentos hacia cada una de sus tesis. Es por ello que al empirismo logicista se le conoce también como atomismo lógico. 
proposiciones matemáticas. Un caso de aplicación matemática como el del parágrafo 15 de la Gramática filosófica, por ejemplo, involucra inferir la proposición:

(1) 'Si tengo 11 manzanas y quiero repartirlas entre algunas personas de tal manera que cada una reciba tres manzanas, puede haber a lo más 3 personas'.

De la operación aritmética

(2)

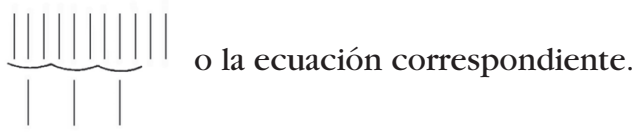

Para el empirista logicista, la proposición (1) es sintética, mientras que la proposición matemática que corresponde al cálculo (2) es analítica. Desde este punto de vista, la aplicación de la matemática implica explicar la manera en que una proposición analítica implica una sintética. Desde el punto de vista de la empirista logicista, tal parece que para resolver problemas prácticos, las proposiciones matemáticas deben "ser verdaderas independientemente de los hechos y también implicar una verdad sobre [...] objetos observables". ${ }^{12}$ Alice Ambrose presenta esta aparente paradoja de la siguiente manera:

¿Cómo puede uno entonces dar cuenta de la armonía entre las dos diferentes áreas de la lógica y los hechos empíricos? ¿Cómo es que podemos aplicar cálculos aritméticos a objetos físicos o cálculo trigonométricos a líneas y ángulos físicos? ¿Hay aquí un misterio genuino o sólo un acertijo gratuito ${ }^{13}$

Lo que el empirismo logicista necesita, entonces, es una teoría de las matemáticas en la que proposiciones sintéticas del tipo de (1) se sigan lógicamente de las proposiciones matemáticas del tipo de (2). Para resolver este problema, el empirismo logicista apela a un mito muy extendido dentro de la

12 Alice Ambrose, "Some Questions in the Foundations of Mathematics", en Stuart Shanker, Ludwig Wittgenstein. Critical Assessments. Volume Three: from the Tractatus to Remarks on the Foundations of Mathematics: Wittgenstein on the Philosophy of Mathematics. Londres, Croom Helm, 1986, p. 204. Publicado originalmente en el Journal of Philosophy, vol. 52, 1955, pp. 197-213.

${ }^{13}$ Idem. "How then can one account for the harmony between the two different areas of logic and empirical fact? How is it that we can apply arithmetical calculations to physical objects, or trigonometrical calculations to physical lines and angles? Is there a genuine mystery here or only a gratuitous puzzle?" 
filosofía de las matemáticas de los últimos cien años: el mito de las matemáticas como ciencia universal. Según este mito, las proposiciones matemáticas son proposiciones universales. Cada teoría matemática define un dominio de aplicación, donde sus proposiciones verdaderas son universalmente válidas. Las verdades de la aritmética, por ejemplo, se aplican a todas las divisiones, sumas, restas, etcétera, posibles e imaginables. A través de esta universalidad, el empirismo logicista explica la amplia aplicabilidad de las matemáticas.

Para los empiristas logicistas, las proposiciones matemáticas tienen un antecedente hipotético implícito que expresa sus condiciones de aplicación. Por ejemplo, $4+4=8$ significa que si "+" es una operación definida sobre la extensión del concepto $F$ tal que si “+” obedece todas las reglas básicas de la operación aritmética de acuerdo a la relación de equivalencia "=", entonces $4 \mathrm{Fs}+4 \mathrm{Fs}=8 \mathrm{Fs}$. De acuerdo a esta posición, la proposición aritmética $4+4$ $=8$ dice que, para todo tipo adecuado de objetos, añadir 4 objetos a un grupo de otros 4 objetos resulta en un nuevo grupo de 8 objetos de ese tipo. Por ejemplo, siendo las manzanas del tipo de objetos a los que se les puede aplicar la aritmética de números naturales, $4+4=8$ implica la proposición más particular de que 'si uno tiene 4 manzanas y las añade a otro grupo de 4 manzanas, uno obtendrá un grupo de 8 manzanas' o, en corto, que ‘ 4 manzanas +4 manzanas $=8$ manzanas'. Para los logicistas este enunciado es una aplicación de $4+4=8$. La principal diferencia entre la proposición sobre manzanas y la proposición matemática pura es que la primera es una hipótesis verificable. Un experimento puede verificar que añadir 4 manzanas a 4 manzanas resulta en 8 manzanas. En general, para los empiristas logicistas, las proposiciones matemáticas de la aritmética de números naturales implican proposiciones genuinas sobre manzanas. La aplicabilidad de la aritmética de números naturales a las manzanas justifica esta inferencia. De ahí la necesidad de incluir una garantía de tal aplicabilidad dentro de la empresa de fundamentación de las matemáticas, según Russell y, más adelante, Ramsey en su obra "Los fundamentos de las matemáticas". ${ }^{14}$

${ }^{14}$ Frank Plumpton Ramsey, "The Foundations of Mathematics", en The Foundations of Mathematics and other Logical Essays. Nueva York, Harcourt, Brace and Co., 1931. Recordemos que el título de la sección de la Gramática filosófica que estamos discutiendo es "La fundamentación de la aritmética en la que ésta se prepara para sus aplicaciones (Russell, Ramsey)" ["Die Begründung der Arithmetik, in der diese auf ihre Anwendungen vorbereitet wird (Russell, Ramsey)"], lo cual revela claramente el sujeto específico de Wittgenstein en esta sección: el proyecto de fundamentación de la aritmética de Russell y Ramsey, en el cual, una fundamentación completa y satisfactoria de la aritmética requeriría especificar y controlar su aplicación a través de una provisión lógica. 


\section{El mito de la universalidad de las matemáticas}

La lógica tiene que ver con el mundo real tanto como la zoología, aunque con sus rasgos más abstractos y generales. ${ }^{15}$

Bertrand Russell

En su Introducción a la filosofía matemática, Bertrand Russell presenta a la lógica, y las matemáticas por extensión logicista, como ciencias de lo más abstracto y general. El origen de este mito es el logicismo que Russell hereda de Frege. Al identificar la verdad matemática con la validez lógica, el logicismo integró la necesidad de las verdades matemáticas con la universalidad de los principios lógicos. Frege mismo ya concebía a las verdades aritméticas como universales y entendía esto como un argumento a favor de su naturaleza lógica. En otras palabras, para Frege, sólo el logicismo podía explicar la universalidad de las matemáticas. En sus Fundamentos de la aritmética, escribió:

Las verdades de la aritmética gobiernan todo lo que es numerable. Éste es el dominio más amplio de todos: ya que a él pertenece no sólo lo real, ni sólo lo perceptible, sino todo lo que podemos pensar. ¿No deberían entonces las leyes aritméticas estar conectadas de manera muy íntima con las leyes del pensamiento ${ }^{16}$

Alentado por los logros de Dedekind y Weierstrass en la aritmetización de las matemáticas, Russell extendió el logicismo de Frege -incluyendo su visión de las matemáticas como universales- hacia la totalidad de las matemáticas. Desde las primeras páginas de Los principios de las matemáticas, Russell caracteriza las proposiciones de las matemáticas (puras) por el hecho de que contienen variables de rango completamente irrestricto, es decir, por ser irrestrictamente universales. ${ }^{17}$ Obedeciendo al dictum aristotélico de que no

15 "Logic is concerned with the real world just as truly as zoology, though with its more abstract and general features» (Bertrand Russell, Introduction to Mathematical Philosophy. Londres, George Allen and Unwin, 1919, p. 169).

16 "Die arithmetischen Wahrheiten beherrschen das gebet des Zählbaren. Dies ist das umfassendste; denn nicht nur das Wirkliche, nicht nur das Anschauliche gehört ihm an, sondern alles Denkbare. Sollten also nicht die Gesetze der Zahlen mit denen des Denkens in der innigsten Verbindung stehen?" (G. Frege, op. cit., parágrafo 14, p. 21).

${ }^{17}$ B. Russell, The Principles of Mathematics. Londres, George Allen and Unwin, 1956, p. 7. 
hay ciencia más que de lo general, Russell reconoció en el método matemático de formalización el mismo proceso de abstracción y generalización que ocurre en el resto de las ciencias, pero llevado a su máxima expresión. Dentro de la filosofía de Russell, las matemáticas eran la ciencia formal por excelencia y, en consecuencia, la ciencia de lo más abstracto y general. ${ }^{18}$

Aún más, la universalidad de las matemáticas se manifestaba en la amplia aplicabilidad de sus resultados, tal y como queda expresado en el siguiente pasaje de Introducción a la filosofía matemática:

Es un principio de todo razonamiento formal el generalizar a lo máximo, ya que así nos aseguramos que un dado proceso de deducción ha de tener aplicaciones más amplias $[\ldots]$

Ciertas características del tema [el cual puede llamarse indistintamente lógica o matemáticas] son claras. Para empezar, en este tema no tratamos con objetos o propiedades particulares: tratamos de manera formal con lo que puede ser dicho de cualquier cosa o cualquier propiedad. ${ }^{19}$

Gracias a su universalidad irrestricta, las matemáticas tienen tantas y tan diversas aplicaciones: del comportamiento social a la física de las partículas elementales.

Por razones filosóficas cuya explicación queda fuera de los objetivos de este artículo, Wittgenstein rechazaba la interpretación empirista logicista de la distinción analítico/sintético, y con ella la mentada universalidad de las matemáticas. A través de su teoría de las matemáticas como gramática, Wittgenstein trata de explicar el fenómeno de la aplicación de las matemáticas sin apelar a su putativa universalidad. En las siguientes secciones presento, primero, el rechazo de Wittgenstein a la universalidad de las matemáticas y, luego, su propuesta de explicación a través de la noción de gramática.

${ }^{18}$ Idem.

19 "It is a principle, in all formal reasoning, to generalise to the utmost, since we thereby secure that a given process of deduction shall have more widely applicable results $[\ldots]$

Certain characteristics of the subject [which may be called indifferently either mathematics or logic] are clear. To begin with we do not, in his subject, deal with particular things or particular properties: we deal formally with what can be said about any thing or any property" (B. Russell, Introduction to Mathematical Philosophy, p. 196). 


\section{Wittgenstein y la aparente generalidad de las matemáticas}

En contraste, para Wittgenstein, las matemáticas no son acerca de todo, sino acerca de nada. Las muchas aplicaciones de las matemáticas no están garantizadas por su generalidad, ya que ellas no son más generales que sus aplicaciones. Para Wittgenstein, las operaciones matemáticas no son generales o abstractas. Por ejemplo, la adición matemática no es una generalización abstracta de todas las adiciones posibles. La proposición aritmética ‘ $4+4=8$ ' no es sobre todas las adiciones. Concierne sólo a la adición aritmética. Es más, el demostrar que las manzanas son objetos del tipo adecuado, si fuera posible, no justificaría el inferir de $4+4=8$, que 4 manzanas +4 manzanas $=8$ manzanas. Añadir el término 'manzanas' a una proposición de aritmética pura no crea una nueva proposición de matemáticas aplicadas.

Pero debemos de tener cuidado de no pensar que " $4+4=8$ " manzanas es la ecuación concreta y $4+4=8$ la proposición abstracta de la cual la primera sólo es un caso especial. De tal manera que la aritmética de manzanas, aunque mucho menos general que la aritmética general, es válida en su propio dominio restringido (para manzanas). No hay tal "aritmética de manzanas", porque la ecuación $4+4=8$ manzanas no es una proposición que trate de manzanas. ${ }^{20}$

Wittgenstein asegura que "no hay tal aritmética de manzanas" en el sentido de que no existe un espacio intermedio entre las matemáticas puras y el mundo real. Para Wittgenstein, las matemáticas constituyen un campo propio, autónomo e independiente. De tal manera que no tiene sentido hablar de proposiciones como ‘ 4 manzanas +4 manzanas $=8$ manzanas' como si pertenecieran tanto a las matemáticas como al lenguaje que utilizamos para hablar del mundo real. ${ }^{21}$

Para entender la aparente generalidad de las proposiciones matemáticas es crucial distinguir entre casos o instancias y aplicaciones. Las proposiciones

20 "Man muß sich aber davor hätten zu glauben “ 4 Äpfel + 4 Äpfel = 8 Äpfel” ist die konkrete Gleichung, dagegen $4+4=8$ der abstrakte Satz, wovon die erste Gleichung nur eine spezielle Anwendung sei. So daß zwar die Arithmetik der Äpfel viel weniger allgemein wäre, als die eigentliche allgemeine, aber eben in ihrem beschränkten Bereich (für Äpfel) gälte. Es gibt aber keine "Arithmetik der Äpfel", denn die Gleichung 4 Äpfel +4 Äpfel = 8 Äpfel ist nicht ein Satz, der von Äpfeln handelt" (L. Wittgenstein, Gramática filosófica, parágrafo 15, p. 604).

${ }^{21}$ Para Wittgenstein, ' 4 manzanas +4 manzanas es $=$ a 8 manzanas' no es una proposición genuina sobre manzanas. Es la proposición matemática ‘ $4+4=8$ ' expresada en términos de manzanas en vez de números. 
matemáticas, en tanto reglas, se aplican. Las generalizaciones genuinas, en contraste, tienen casos o instancias. La diferencia es importante porque, mientras que entre generalización e instancia hay una relación de consecuencia lógica (toda proposición general implica lógicamente a sus instancias particulares), ninguna relación lógica de este tipo existe entre regla y aplicación.

Considere las siguientes cuatro proposiciones:

(4) $a+(b+c)=(a+b)+c$

(5) $(3+4)+6=3+(4+6)$

(6) Todos los camellos son herbívoros.

(7) Mi camello es herbívoro.

(5) es una aplicación de (4), mientras que (7) es una instancia de (6). La verdad de generalizaciones genuinas como (6) descansa en una inducción de casos particulares como (7). La verdad de proposiciones gramáticas como (4) no depende de ninguna manera de la verdad de proposiciones como (5). De ninguna manera (5) confirma (4). Aun si la proposición (5) ocurriera en la prueba de (4), no lo haría como instancia confirmadora. Es por eso que la inducción matemática es tan distinta de la inducción genuina tal y como aparece en las ciencias no formales. En las matemáticas no existen las instancias o los casos. Por lo tanto, no se puede confirmar ninguna generalización. Es erróneo caracterizar a las proposiciones matemáticas por su generalidad. La proposición (5) no es menos general que (4), aun cuando parezca que (4) es sobre todos los números y (5) es sobre unos en particular. ${ }^{22}$

Refutar el mito de que proposiciones matemáticas como (4) son más generales que proposiciones como (5) requiere re-interpretar la cuantificación y el papel de las letras llamadas variables en las matemáticas. Esto es lo que Wittgenstein se propone en las secciones XIII y XIV de la segunda parte de la Gramática filosófica. En el parágrafo 150, Wittgenstein describe las tres funciones posibles de las letras en las matemáticas: 1) como constante general,

${ }^{22}$ Para Wittgenstein, en matemáticas, es imposible generalizar a partir de casos particulares. De una serie de verdades matemáticas conocidas es imposible generalizar para casos similares. El hablar de casos similares implica clasificar las proposiciones bajo un concepto general. Sin embargo, para Wittgenstein, tampoco hay conceptos generales en las matemáticas. Los conceptos matemáticos no son generalizaciones, sino disyunciones. Para Wittgenstein, agrupar proposiciones matemáticas bajo un concepto matemático no resultaría en una proposición matemática general, sino en la mera conjunción de las proposiciones originales. Una conjunción no es más general que cualquiera de sus conyuntos. Por lo tanto el agrupar proposiciones matemáticas bajo un concepto matemático no resulta en una proposición matemática más general. El enunciado matemático resultante no es ni menos ni más general que sus partes. 
2) como incógnita y 3) como marca de un espacio en blanco. En el primer caso, la letra pertenece al lenguaje del sistema de cálculo. En los otros dos casos, es un elemento externo. De cualquier manera, cada letra en un enunciado de cálculo es una constante general. ${ }^{23}$ En la primera nota al parágrafo150 de las Observaciones filosóficas, Wittgenstein escribió: "Pero este signo '(x)' dice exactamente lo opuesto de lo que dice en casos no matemáticos... Es decir, precisamente que debemos tratar a las variables en la proposición como constantes". ${ }^{24}$

Para Wittgenstein, las letras tienen significados radicalmente diferentes en las notaciones matemática y lógica. Ignorar esta diferencia produce la idea equivocada de que las variables pueden ocurrir no ligadas. Las fórmulas lógicas representan la forma lógica de proposiciones genuinas. Dado que las proposiciones genuinas pueden ser generales, es necesario poder expresar la generalidad en el formalismo lógico. Tal es la función de las letras en el formalismo lógico. Sin embargo, las fórmulas matemáticas por sí solas no representan la forma lógica de ninguna proposición genuina. ${ }^{25}$ En consecuencia, sus letras tienen un papel distinto. En matemáticas, el hablar sobre todos los números, por ejemplo, sugiere que las proposiciones matemáticas son generales, pero la generalidad de las matemáticas es de otro tipo. La generalidad matemática combina totalidad y necesidad en una sola noción modal. "Porque en matemáticas 'necesario' y 'todo' van juntos. (A menos que reemplacemos estas expresiones idiomáticas por completo por unas que sean menos confusas)". ${ }^{26}$ La modalidad matemática no establece lo que es posible o necesario, ni lo general o particular, sino lo que tiene sentido o no.

De acuerdo al análisis de Wittgenstein, una fórmula matemática tiene tres posibles interpretaciones dependiendo del papel sintáctico de sus letras. Esta clasificación corresponde a tres posibles preguntas que pueden hacerse acerca de tales expresiones: ${ }^{27}$

${ }^{23}$ Para Wittgenstein, un enunciado de cálculo es aquel que conecta un cálculo con su resultado. Las ecuaciones aritméticas, como ' $3+7=10$ ', son enunciados de cálculo porque conectan cálculos como la adición de $3+7$, y su resultado, el número 10. $C f$. Axel Barceló, Mathematics as Grammar. 'Grammar' in Wittgenstein's Philosophy of Mathematics during the Middle Period. Tesis. Bloomington, Universidad de Indiana, 2000.

24 "Dieses Zeichen '( $x$ )' sagt aber gerade das Gegenteil dessem, was es in den nicht mathematischen Fällen sagt [...] nämlich gerade, daß wir die Variable in dem Satz als Konstante auffassen sollen" (L. Wittgenstein, Gramática filosófica, parágrafo 150, nota 1, p. 164).

${ }^{25}$ Sin embargo, Wittgenstein no dice si las fórmulas matemáticas pueden formar parte de fórmulas lógicas que sí lo expresen, como lo sostiene Ramsey en sus Fundamentos de las matemáticas.

${ }^{26}$ L. Wittgenstein, Observaciones filosóficas, parágrafo 150.

${ }^{27}$ Idem. 
1. Si todas sus letras son constantes en el sistema de cálculo, la fórmula es un enunciado del cálculo. Un caso límite es aquel en el que no ocurre ninguna letra. Para estas expresiones, tiene sentido preguntar si la fórmula es correcta o no. En el caso de ecuaciones aritméticas, por ejemplo, la respuesta depende de si las reglas del sistema de cálculo permiten que las expresiones numéricas a ambos lados del símbolo "=" se transformen en la misma expresión. ${ }^{28}$ Por ejemplo, la ecuación " $3+4=7$ " es un enunciado correcto, porque las reglas de la adición de números naturales permiten transformar la expresión " 3 + 4" en el numeral " 7 ". En este caso, se dice que 7 es el resultado de sumar 3 y 4 .

2. Si por lo menos una de las letras expresa una incógnita y ninguna otra marca un espacio en blanco, entonces la fórmula no es un enunciado sino un esquema. En este caso, no tiene sentido preguntar si es correcta o no. Pero sí tiene sentido preguntar si tiene solución y cuál es ésta. Una ecuación de este tipo tiene solución solamente si es posible reemplazar la o sus incógnitas por constantes generales (no necesariamente letras) para producir un enunciado verdadero. Por ejemplo, la fórmula " $3+4=x$ " no es un enunciado de la aritmética de números naturales porque incluye una letra que no pertenece al vocabulario de ese sistema de cálculo. La letra " $x$ " no es un símbolo aritmético ni un numeral. Expresa una incógnita. La incógnita puede ser sustituida por símbolos de la aritmética -numerales en particular-, y producir ecuaciones del primer tipo, es decir, enunciados de cálculo. Sustituir la " $x$ " por el numeral " 3 " en el ejemplo anterior resulta en la fórmula " $3+4=3$ ". Sustituirla por “35" da " $3+4=35$ ", etcétera. Una de estas sustituciones - " $x$ " por " 7 " - resulta en una fórmula correcta - " $3+4=7$ ". Esto significa que la ecuación original tiene solución.

3. Si por lo menos una de las letras marca un espacio en blanco (leere Stelle), entonces la expresión está incompleta. En este caso, sólo tiene sentido preguntar si la expresión es permitida por la sintaxis del sistema de cálculo. Una expresión incompleta es sintácticamente permitida si es posible construir una fórmula bien formada con tan solo llenar sus espacios en blanco. Por ejemplo, la expresión " $3+x=7$ " puede ser una expresión incompleta si se toma la letra " $x$ " como marca de un lugar vacío. Al igual que el ejemplo anterior, la existencia de la ecuación " $3+4=7$ " garantiza que la expresión " $3+x=7$ " es sintácticamente permitida, dado que su espacio en blanco puede ser así llenado para producir una fórmula bien formada.

Wittgenstein adopta esta clasificación para combatir el mito de que las matemáticas tienen diferentes niveles de generalidad. Expresiones con letras no son más generales que expresiones con otras constante matemáticas, como

${ }^{28}$ Ibid., parágrafo 154. 
los numerales. En una nota a pie de página del parágrafo 150 de las Observaciones filosóficas, Wittgenstein escribe: "Aún no he enfatizado lo suficiente que $25 \times 25=125$ está precisamente al mismo nivel y es precisamente del mismo tipo que $x^{2}+y^{2}+2 x y=(x+y)^{2} .^{29}$

Wittgenstein ilustra esta distinción con la expresión " $a+(b+c)=(a+b)$ $+c$ ". Dado que las letras son parte del vocabulario algebraico, mas no del aritmético, esta fórmula puede expresar tanto una proposición algebraica como la ley de la asociatividad para la adición aritmética. En álgebra, las letras son constantes generales. En aritmética, sólo pueden ocurrir como incógnitas o marcas de espacios vacíos. La ley de la asociatividad aditiva de la aritmética " $a+(b+c)=(a+b)+c$ " parece expresar una propiedad general de todos los números o todas las adiciones. Sin embargo, no es así, ya que no es una proposición general, sino un esquema.

\section{Wittgenstein y la aplicación de las matemáticas}

La posición de Wittgenstein empieza a distinguirse de la de los logicistas desde la interpretación misma del problema no matemático. Leyendo con más cuidado el mentado pasaje de la Gramática filosófica (parágrafo 15 de la parte II, o parágrafo 111 de las Observaciones filosóficas), el lector puede distinguir tres elementos:

(8) El problema práctico (Aufgabe): "Si tengo 11 manzanas y quiero repartirlas entre algunas personas de tal manera que cada una reciba tres manzanas, ¿̨cuántas personas puede haber?” La pregunta es hipotética y modal, en tanto que pregunta qué es posible. Pregunta cuál es el número de personas a las cuales es posible dar 3 manzanas de un grupo de 11.

(9) La solución (Lösung): Wittgenstein deja en claro que la solución del problema es el número 3 , no que puedan haber tres personas.

(10) La predicción (Vorhersagung) de que podría darle a tres personas su porción de 3 manzanas y quedarían 2. Ésta sí es una predicción genuina y verificable, no matemática.

En el pasaje ya mencionado, Wittgenstein explícitamente dice que el cálculo suministra (liefert) la solución (9) al problema práctico (8). No nos autoriza (berechtigen) hacer la predicción (10). La diferencia es esencial para

29 "Ich habe noch zu wenig betont daß 25 x $25=625$ auf genau derselben Stufe und von genau derselben Art ist wie $x^{2}+y^{2}+2 x y=(x+y)^{2^{\prime \prime}}$ (L. Wittgenstein, Gramática filosófica, parágrafo 150, nota 4, p. 164). 
entender la naturaleza gramática de la aplicación de las matemáticas. Si bien el cálculo suministra la solución, no justifica la de problemas prácticos. Dar solución a problemas no matemáticos es muy distinto que justificar una predicción no matemática. Wittgenstein acepta que en su ejemplo, cálculo y ecuación son idénticos, pero éste no predice que, una vez distribuidas, "quedarán 2 manzanas". Pero sí dice que es posible distribuir 11 manzanas entre 3 personas de tal manera que cada una reciba 3 manzanas. Sin embargo, esta última no es una predicción física, sino una proposición gramática.

El cálculo dice que es posible distribuir 11 manzanas entre 3 personas de tal manera que cada una reciba 3 manzanas. La pregunta "Si tengo 11 manzanas y quiero repartirlas entre algunas personas de tal manera que cada una reciba tres manzanas, ¿cuántas personas puede haber?” es gramática. La modalidad a que hace referencia cuando pregunta por el número máximo de personas posibles no es una posibilidad física, sino gramática. "Porque la palabra 'puede' en ese enunciado no indica una posibilidad física (fisiológica, psicológica)".$^{30}$ La relación entre el cálculo matemático y los 3 elementos antes mencionados queda claro una vez que se toma en consideración que, para Wittgenstein, las proposiciones matemáticas son reglas gramáticas.

La respuesta adecuada a la pregunta en (8) "Si tengo 11 manzanas y quiero repartirlas entre algunas personas de tal manera que cada una reciba tres manzanas, ¿cuántas personas puede haber?” no es un enunciado general sobre manzanas y su distribución. Es una respuesta gramática, es decir matemática. Su respuesta apropiada debe ser una regla gramática. El cálculo matemático suministra esta regla. La respuesta que da el cálculo es adecuada porque la pregunta es gramática. No pregunta por las propiedades necesarias de las manzanas o de su distribución, sino sobre qué tiene sentido predecir. La respuesta es una regla para el uso de la palabra 'manzana'. Esta regla dice que la proposición genuina (11) "le doy a 3 personas su ración y quedan 2 manzanas" es gramáticamente correcta, ya que este enunciado es una instancia de aplicación de la regla dada por el cálculo. Debemos recordar también, que dentro de la teoría gramática de Wittgenstein durante estos años, que un enunciado del lenguaje no-matemático sea gramaticalmente correcto significa que lo que en él se dice es posible. En este caso en particular, que (11) sea gramáticamente correcto significa que es posible darle a 3 personas su ración y que queden 2 manzanas. La corrección gramática de (11) le da sentido a la predicción.

30 "Denn das Wort "kann" in diesem Satz deutet nicht auf eine physiche (physiologische, psychologishe) Möglichkeit" (L. Wittgenstein, Gramática filosófica, parágrafo 14, p. 596). 
Para Wittgenstein, la predicción sobre manzanas y su distribución no es la solución del problema, sino la aplicación de tal solución. La predicción (10) no es la solución al problema práctico (8). La resolución del problema matemático precede la del no-matemático. La solución matemática es anterior a la predicción física. La formulación de la predicción requiere el cálculo matemático, porque es su aplicación. El cálculo se aplica al formular la predicción. El cálculo suministra la gramática de la proposición genuina que expresa la predicción física. La formulación de la predicción involucra la aplicación del cálculo como regla gramática. Sólo en este sentido se aplica el cálculo matemático a la solución de problemas no matemáticos. Aplicar un cálculo matemático significa usarlo como regla gramática para la construcción o transformación de proposiciones. En el parágrafo 107 de las Observaciones filosóficas, Wittgenstein dice:

Las proposiciones aritméticas, como las tablas de multiplicación y similares, o también como definiciones que no tienen proposiciones enteras a ambos lados, se usan en aplicación a proposiciones. Y de cualquier manera ciertamente no las puedo aplicar a nada más. (Por lo tanto no necesito primero describir su aplicación). ${ }^{31}$

La aplicación de un cálculo matemático a proposiciones externas requiere insertar las reglas de un sistema de cálculo en la gramática del otro. En particular, la aplicación de un cálculo matemático a una proposición genuina requiere insertar las reglas del sistema de cálculo en la gramática del lenguaje natural. De esta manera, las reglas de cálculo se convierten en reglas gramáticas del lenguaje natural. Para Wittgenstein, el cálculo no es sólo una regla en la gramática de los números naturales, sino también una regla gramática del lenguaje natural. ${ }^{32}$ Una de sus aplicaciones es la construcción de enunciados del español como "podría darle a 3 personas su ración y quedarían 2 manzanas". De acuerdo a las notas de Friedrich Weissmann, durante una conversación en la casa de Schlick, el 28 de diciembre de 1930, Wittgenstein dijo:

¿Qué significa aplicar un sistema de cálculo? [...] El sistema de cálculo se aplica de tal manera que se proporcione la gramática de un lengua-

31 "Die arithmetischen Sätze dienen, wie Multiplikationstabellen und dergleichen, oder auch wie Definitionen, auf deren beiden Seiten nicht ganze Sätze stehen, zur Anwendung auf die Sätze. Und auf etwas anderes kann ich sie ja sowieso nicht anwenden. (Ich brauche also nicht erst irgendwelche Beschreibung ihrer Anwendung)" (L. Wittgenstein, Observaciones filosóficas, parágrafo 107).

${ }^{32}$ En sentido estricto, los cálculos aritméticos son reglas gramáticas de cualquier lenguaje natural que contenga expresiones numéricas. 
je. Porque, lo que las reglas permiten o prohíben, corresponde en la gramática a las palabras 'sentido' y 'sin sentido'. ${ }^{33}$

En el ejemplo del parágrafo 15, el cálculo (2) da la gramática de la proposición genuina sobre manzanas (11). Con esta proposición se hace la predicción física. El cálculo hace la predicción posible, pero su verdad permanece independiente de las matemáticas. Requiere verificación. El cálculo en sí mismo no predice nada sobre las manzanas.

En el parágrafo 17 de la Gramática filosófica, Wittgenstein escribe: "Un enunciado basado en un cálculo erróneo (como 'cortó una tabla de 3 metros en 4 partes de 1 metro cada una') no tiene sentido y eso ilumina lo que significa 'tener sentido' y 'significar algo con un enunciado' ". 34

La aplicación de un cálculo correcto resulta en un enunciado gramáticamente bien formado. La aplicación de un cálculo incorrecto resulta en un sin-sentido. Si el cálculo es correcto como en el ejemplo del parágrafo 15, el enunciado tiene sentido. Si el cálculo es incorrecto, como es el caso en el parágrafo 17, el enunciado no tiene sentido. En ambos casos el cálculo no verifica la proposición. El cálculo matemático no tiene que ver nada con la verdad o falsedad de su aplicación. No la justifica. Un cálculo correcto puede producir una proposición genuina falsa, tanto como una verdadera. La verdad de la proposición no matemática resultante de la aplicación del cálculo es independiente de éste.

La aplicación de las matemáticas, como la aplicación de cualquier regla, no es un fenómeno lógico ni epistemológico. Si bien cada vez que se aplica un cálculo matemático a la solución de un problema práctico se establece una conexión entre el espacio de lo matemático y el espacio de lo no-matemático, esta conexión no se establece, tal y como lo creían los empiristas logicistas, a través de relaciones inferenciales o de justificación. La relación entre cálculo matemático y predicción física ni es lógica (la aplicación no se sigue de la regla) ni epistemológica (la regla no justifica su propia aplicación). Apelando a la noción de regla gramática, Wittgenstein puede explicar la aplicación de las matemáticas sin adscribirle universalidad a las proposiciones matemáticas. De esta manera, no sólo distingue aplicabilidad de universalidad, sino que demuestra que, en el caso de las matemáticas, como el de cualquier sistema de reglas, se puede tener la una sin la otra.

33 "Was heißt es, einen Kalkül anwenden?... Man wendet den Kalkül in der Weise an, daß er die Grammatik einer Sprache ergibt. Dem, was die Regel erlaubt oder verbietet, entspricht dann in der Grammatik das Wort 'sinnvoll' und 'sinnlos'“.

34 "Ein Satz, der auf einer falschen Rechnung beruht (wie etwa 'er teilte das $3 \mathrm{~m}$ lange Brett in 4 Teile zu je 1 m') ist unsinnig und das beleuchtet, was es heißt 'Sinn haben' und 'etwas mit dem Satz meinen'". 